\title{
The Colored Oil Property Effect on Switching Behavior of Electro-Fluidic Display
}

\author{
Yuanyuan Guo',2, Yong Deng2,3, Biao Tang1*, Alex Henzen1, Guofu Zhou',2,3 \\ ${ }^{1}$ Guangdong Provincial Key Laboratory of Optical Information Materials and Technology \& Institute of Electronic Paper Displays, \\ South China Academy of Advanced Optoelectronics, South China Normal University, Guangzhou, China \\ ${ }^{2}$ Shenzhen Guohua Optoelectronics Tech. Co. Ltd., Shenzhen, China \\ ${ }^{3}$ Academy of Shenzhen Guohua Optoelectronics, Shenzhen, China \\ Email: yuanyuan.guo@guohua-oet.com, ^tangbiao@scnu.edu.cn
}

How to cite this paper: Guo, Y.Y., Deng, Y., Tang, B., Henzen, A. and Zhou, G.F. (2020) The Colored Oil Property Effect on Switching Behavior of Electro-Fluidic Display. Materials Sciences and Applications, 11, 167-176.

https://doi.org/10.4236/msa.2020.112010

Received: January 9, 2020

Accepted: February 16, 2020

Published: February 19, 2020

Copyright $\odot 2020$ by author(s) and Scientific Research Publishing Inc. This work is licensed under the Creative Commons Attribution International License (CC BY 4.0).

http://creativecommons.org/licenses/by/4.0/ Open Access

\begin{abstract}
The switchable oil layer driven by electrowetting gives visible color and light valve control, which is the basis of Electro-Fluidic Displays. The colored oil's property is a key factor that influences the Electro-Fluidic Displays switching behavior. A purple oil was formulated by the oil-soluble purple dye in decane in this study. The dye molecule itself is nonpolar and it doesn't dissolve in water. The concentration of colored oil influenced the oil/water interfacial tension and oil viscosity. The relationship of EFD switching behavior with oil/water interfacial tension, oil viscosity, and oil conductivity has been systematically investigated. The oil/water interfacial tension decreased with increasing oil concentration, in the meanwhile, the conductivity increased. Oil conductivity was one of the key factors that influenced the Electro-Fluidic Displays optical property. We found for the first time that at the lower oil concentration $(2 \%-10 \%)$, the interfacial tension plays a main role effect on the rupture voltage and response time, but as the conductivity of higher concentration of colored oil increased (at 20\%), the rupture voltage-controlled both by conductivity and interfacial tension.
\end{abstract}

\section{Keywords}

Electro Fluid Display Device, Oil/Water Interfacial

Tension/Viscosity/Conductivity, Rupture Voltage, Opening Ratio

\section{Introduction}

The electrowetting phenomenon was first described in 1875 by Lippmann [1]. Nowadays, a hydrophobic insulating layer on the electrode is normally used to reduce contact angle hysteresis to get better electrowetting behavior [2]. By 
changing the electric field across the insulating layer, the contact angle of liquid/dielectric solid surface changes. Theoretically, the contact angle of a water drop decreased by increasing the electric field, but because of free charge aggregation at the three-phase contact line, the water contact angle can never be zero [3] [4]. Electrowetting has application in lab-on-chip for micro-fluid manipulation [5] [6], optical lens systems [7] [8], optical fibers [9], and displays [10] [11]. Figure 1(a) demonstrates the basic structure and principles of electro-fluid display cells.

For the electro-fluid display application, the colored oil layer acts as an optical valve which was stabilized in the pixel due to the capillary and hydrophobic force (Figure 1(b)). The gravitational force can be neglected on this small-scale $(<100 \mu \mathrm{m})$. Without voltage, the colored oil forms a thin film between the hydrophobic insulator surface and the water, since the interfacial tension between the water/insulator pair is larger than the interfacial tension between the insulator/oil and oil/water layers. The colored oil layer can then be switched on and off with an electric field. Once the voltage is applied, the equilibrium stage is disturbed by the static electric force and the oil film subsequently breaks and shrinks to one corner of the pixel, thus reducing the free energy [12]. The response time can reach less than $10 \mathrm{~ms}$, which provides an advantage over other reflective display technologies that video frame rates are possible. Thibault et al. reported the interfacial tension effect on electrowetting driven motion. They concluded that the driving voltage decreases with increasing dye concentration and the interfacial tension plays a significant role in the speed of the oil movement in a large structure [13]. Although the dye used for electro-fluid display should be nonpolar, yet organic molecules still can be polarized under a high electric field, resulting in increased oil conductivity. A research group of Samsung discovered the "flow back" defect. They found that the dyed oil film in the pixel tends to reduce the contraction even if the voltage is maintained [14]. They discussed that this flow back effect is mainly dependent on the structure of the dye and that more symmetrical molecules provide shorter flow back time. But
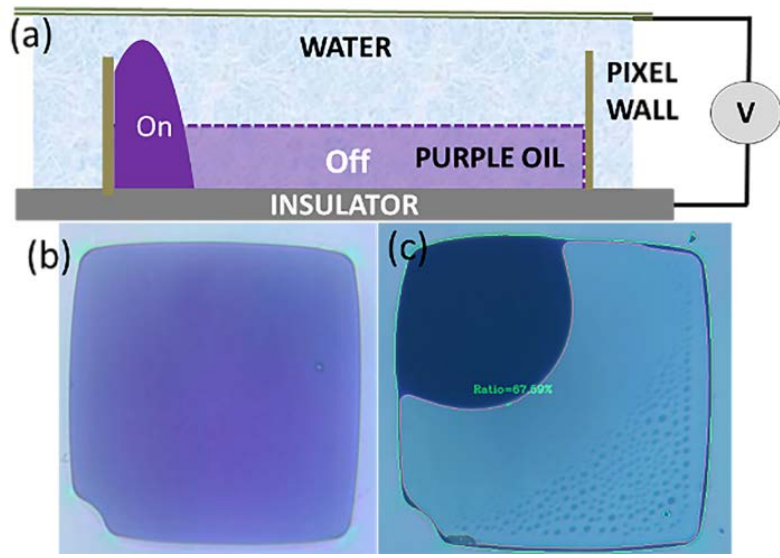

Figure 1. The composition of an electro-fluid display (a): on and off-state; (b): a filled single pixel for testing in this study and (c) schematic of opening ratio calculation. 
they did not consider the variation of the colored oil's conductivity which also relates to the fundamental property of dye molecules. The papers published in our group recently showed that the dyes with a tertiary amino group can be protonated at the oil/water interface that will affect the electrowetting contact angle [15] [16]. In this work, we chose decane as the solvent because of its high dielectric constant, low viscosity and solubility in water. We investigated the relationship of the colored oil/water interfacial tension, colored oil viscosity, and conductivity of different oil concentrations and surfactant concentrations with the effect on electro-fluid display cell rupture voltage and switching behavior.

\section{Electro-Fluid Display Cell Fabrication and Experimental Methods}

The electro-fluid display cell under test was produced as previously reported from our group, using a $5.4 \mu \mathrm{m}$ high pixel wall and $150 \mu \mathrm{m} \times 150 \mu \mathrm{m}$ square pixel. Amorphous fluoropolymer (AF1600, Chemours, cr 1.934) was spun coated onto ITO glass to form an $850 \mathrm{~nm}$ hydrophobic insulator. A descum process, using reactive ion etching, was applied after lithography of the pixel wall to make sure no residue was left on the hydrophobic insulator surface, and a $640 \mathrm{~nm}$ fluoropolymer film was left after all production process. A nonpolar oil-soluble anthraquinone purple dye (purity $\geq 98 \%$ ) was purchased from a local company (Jiaxing Evershine Chemical Co. Ltd.). 2\%, 5\%, 10\% and 20\% dye concentrations were formulated in decane (Aldrich, $\geq 99 \%$ ) (dielectric constant $\varepsilon$ r 2). Water for interfacial tension measurement was double distilled. The colored oil was then filled into the pixels between water and hydrophobic insulating layer with the method described earlier [17].

Oil/water interfacial tension was determined by the pendant drop technique with the Detaphysics Contact Angle System (OCA 15Pro) at 25 [18]. A 1.67 $\mathrm{mm}$ diameter hook shape $\mathrm{U}$ tube syringe needle was applied for oil/water interfacial tension measurement because the density of oil is smaller than water [19]. Since the colored oil is not transparent, the interfacial tension was monitored in real-time, and the interfacial tension was determined when the data curve reached a plateau value. A testing cell with a $22 \mu \mathrm{m}$ gap between two ITO electrodes on glass was made, and the cell was filled with colored oil by capillary force. A DC voltage was applied between the ITO electrodes, and the electric current was recorded by a Picoammeter (Keithley 6487) at $-35 \mathrm{~V}$ for $25 \mathrm{~s}$ to compare the conductivities of oil. A lab-view program was utilized to record the current data. The same test cells were used to keep the gap between testing constant, and the cell was cleaned by petroleum ether and dried at 110 degrees each time after testing. Before testing, the filled cell was shorted for $120 \mathrm{~s}$. Luminance was monitored on $-45 \mathrm{~V}$ with $5 \mathrm{~Hz}$ ( $50 \%$ duty cycle) by a colorimeter (Admesy ages $\left.-45^{\circ}\right)$. The luminance was calculated into reflectance using the experienced equation $R=0.058 L+0.913$. The visual response time was measured as the time from voltage-on until $90 \%$ reflectance is reached. The pixel was driven by a DC voltage cycle, from $0 \mathrm{~V}$ to $-52 \mathrm{~V}$ back to $0 \mathrm{~V}$, a video was recorded under a mi- 
croscope. A homemade program automatically calculated the white area ratio (WAR $=$ Open area/pixel area) using a proprietary routine in Visual Studio 2012.

\section{Result and Discussion}

The oil layer in the electro fluid display device has to have absorption equals to 1 for $5 \mu \mathrm{m}$ thickness, the normal concentration for display purpose in our lab was $10 \%$. The oil with concentration below $2 \%$ was colorless while due to the limitation of purple dye's solubility $20 \%$ was the maximum concentration. As purple dye concentration increased from $2 \%$ to $20 \%$, the interfacial tension dropped linearly from $42 \mathrm{mN} / \mathrm{m}$ to $23 \mathrm{mN} / \mathrm{m}$. In the meanwhile, the viscosity increased from $0.4 \mathrm{mPas}$ to $1.4 \mathrm{mPas}$ (Figure 2(a)). The electrowetting follows the Young-Lippman equation, where the rupture related to oil/water interfacial tension, insulator's dielectric constant and thickness. In the equation, the liquid conductivity, especially the insulator oil's conductivity was not taken into consideration. We found that as the oil concentration increased, the oil's conductivity changed. Even though liquid conductivity is customarily measured using AC at a specific frequency because liquid tends to form double-layers under a DC voltage. But for electro-fluid display devices, a DC voltage was used due to the presence of dielectric material. We used a homemade cell to measure the conductivity of different concentration oils. The current through the $22 \mu \mathrm{m}$ thick oil layer between two ITO electrodes was recorded at $-35 \mathrm{~V}$. The current through the oil layer indicates the changing of the oil conductivity. The following equation calculated the conductivity:

$$
\rho=I \cdot d / V \cdot A(\mathrm{~S} / \mathrm{m})
$$

$I$ is the measured current, $d$ is the distance between two ITO electrode, $A$ is the area of the electrode, $V$ is the voltage.

As shown in Figure $2(\mathrm{~b})$, the conductivity of pure decane was $3.5 \times 10^{-12} \mathrm{~S} / \mathrm{m}$. The conductivity of $2 \%$ and $5 \%$ dye solution increased from $5.7 \times 10^{-12} \mathrm{~S} / \mathrm{m}$ to $1.4 \times 10^{-11} \mathrm{~S} / \mathrm{m}$. The $20 \%$ oil solution's current increased with time under $-35 \mathrm{~V}$ which indicates a strong polarization state of the dye molecules in the solution and the conductivity increased quickly to $1.1 \times 10^{-10} \mathrm{~S} / \mathrm{m}$. The dye molecule might be protonated under the high voltage, and the oil layer thus may have become more conductive compared to low concentration. The maximum conductivity at $-35 \mathrm{~V}$ DC depends exponentially on the dye concentration.

The flow back phenomenon was first observed by R. Massard [20]. Flow back is the tendency of the pixel aperture and display brightness diminishing under continued addressing. Flow back also affects the switching speed and the power consumption of electro-fluidic displays [14]. In the past, it was found that the flow back has a strong dependence on the concentration of dye and dye's chemical nature. Recently, Li et al. found that the dye with the amino group can be protonated result in changing the contact angle on the dielectric surface [16]. 

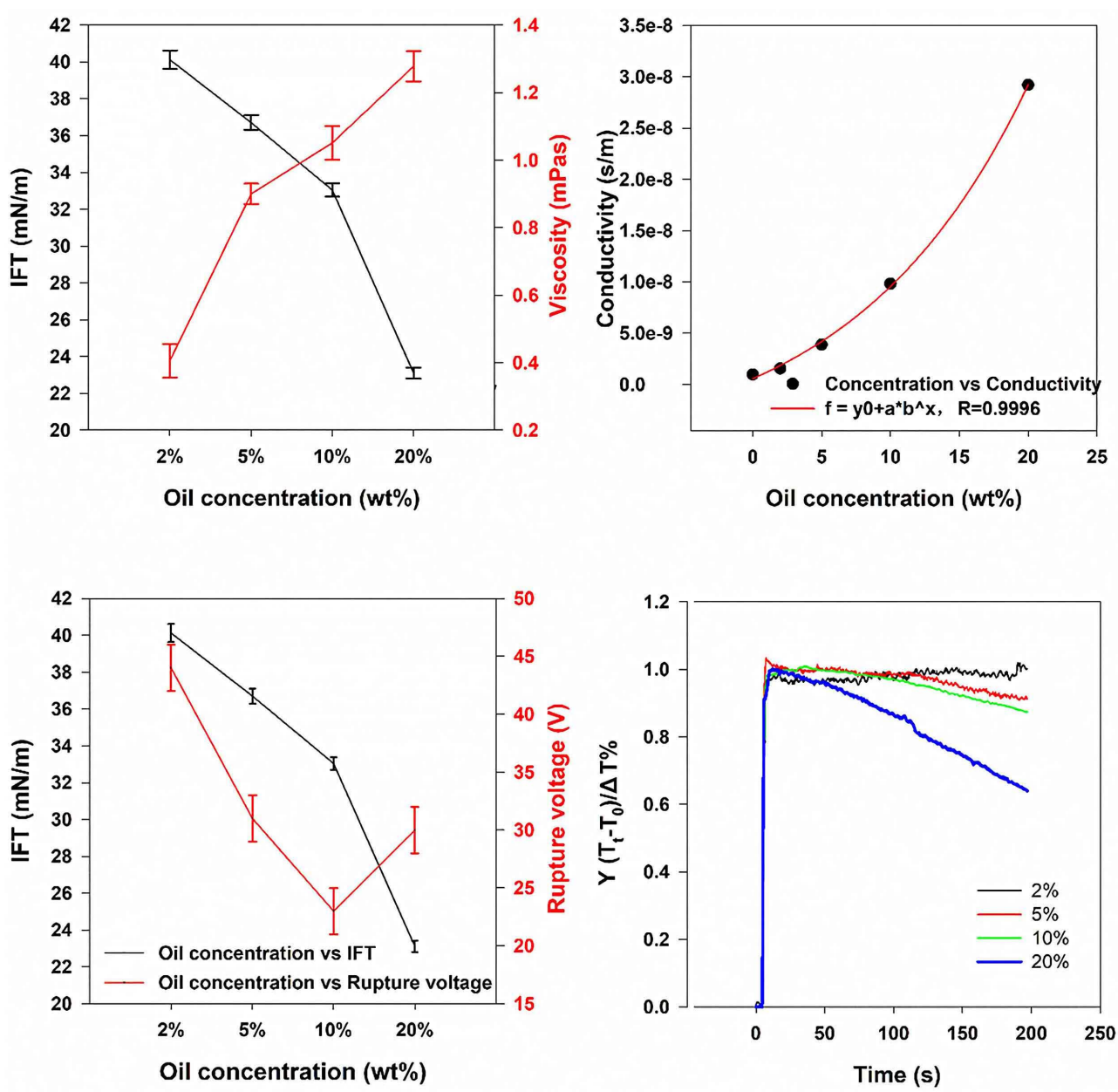

Figure 2. (a) The interfacial tension and viscosity relation with different oil concentration; (b) the conductivity changes of different concentration oil solution; (c) The relationship of rupture voltage with interfacial tension and oil conductivity; (d) Comparison of transmittance $\left(\left(T_{t}-T_{0}\right) / \Delta T\right)$ with time.

Here, we investigated the effect of colored oil conductivity on the flow back properties of the electro-fluidic display device.

The transmissivity was monitored by fiber optic spectrometer after the pixel opened at $-45 \mathrm{~V}$. Since the transmittance through different dye concentration oil layers was different, $\left(T_{t}-T_{0}\right) / \Delta T$ was used to normalize the deviation. Because charges will transfer from the oil/water interface to the dielectric insulator surface result in the colored oil's conductivity changes with time. The higher the conductivity of oil the faster the change transfer process and the accumulation of charges on the dielectric surface. The electrostatic force is reduced due to charge neutralization on the contact line area and the oil drop flows back slowly into the pixel area, and eventually closes entirely, even with the voltage applied (Figure 3 ). As a result, the transmittance through pixels will decrease with time. The slope in Figure 2(d) indicates the velocity of flow back speed, and it shows the flow back occurs more rapidly with increasing dye concentration. The pure decane was also tested to compare the flow back time, but because decane is transparent, 


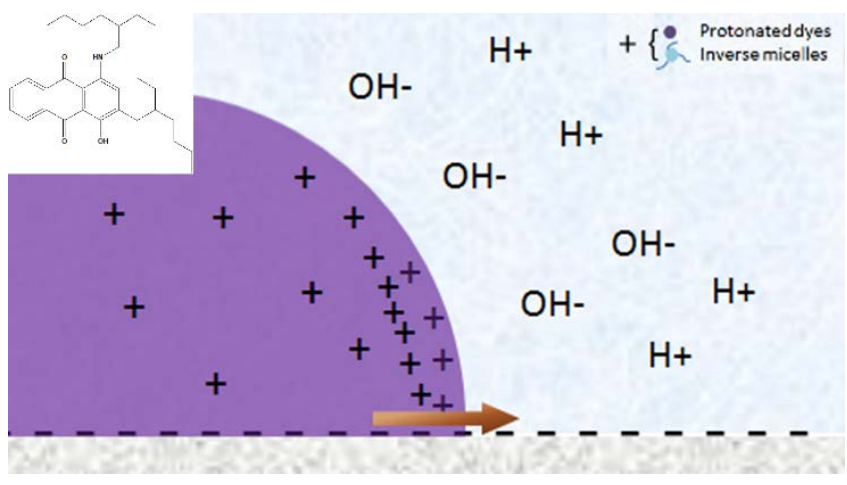

Figure 3. Schematic diagram of the flow back phenomenon (the + presents the protonated dye or charged inverse micelles), the insert picture shows the purple dye structure.

this cannot be monitored by measuring transmissivity. The flow back time was observed under a microscope, and no apparent flow back occurred within $5 \mathrm{mi}-$ nutes. The colored oil-filled pixel was switched between $0 \mathrm{~V}$ to $-52 \mathrm{~V}$ at a rate of $-1 \mathrm{~V} / 0.3 \mathrm{~ms}$ rate, and the video was recorded under a microscope. The white area ratio was then measured from this video using a proprietary program in visual studio. As the applied voltage increased, at a specific value the pixel opened, and the white area ratio suddenly enlarged, and this voltage was determined as the "rupture voltage" (Figure 4). The opening speed is reduced after the white area ratio reached about $55 \%$ for all test oil solution. After $55 \%$ opening ratio, the open area is increased linearly until reaching the largest percentage at $-52 \mathrm{~V}$. As Figure $4(\mathrm{a})$ shows, the maximum opening ratio increased from $60 \%$ to a maximum of $74 \%$ with $2 \%$ and $5 \%$ dye concentration, respectively. The maximum white area ratio dropped to $68 \%$ and even lower to $64 \%$ with $10 \%$ and $20 \%$ dye concentration which can be explained that as the conductivity increased under applied voltage the oil layer needs a higher potential to reach the same opening ratio. The rupture voltage decreased with the oil concentration of $2 \%$, $5 \%$ and $10 \%$ due to the reduced oil/water interfacial tension. Only in one case when the concentration reached $20 \%$ instead of further decrease the rupture voltage increased to $30 \mathrm{~V}$ (Figure 4(a)). This suggested that at lower dye concentrations the conductivity did not determine the rupture voltage compared with oil/water interfacial tension. Only at a higher concentration (over 10\%), the conductivity was one of the leading factors determining the rupture voltage together with interfacial tension, and thus a higher voltage was needed to reach a large opening ratio with a high conductivity oil solution.

According to the literature [21], the rupture voltage can be simulated by the following equation:

$$
V=\left(2 \pi^{2} \gamma / C^{\prime \prime}(h) L_{X}^{2}\right)^{1 / 2}
$$

$C(h)$ is the capacitance of an oil film of thickness $h, \gamma$ is the oil/water interfacial tension, $L x$ is a short lateral dimension.

But this equation does not take oil conductivity into account and also there was not a discourse about the mechanism of charge transfer within the oil phase. 

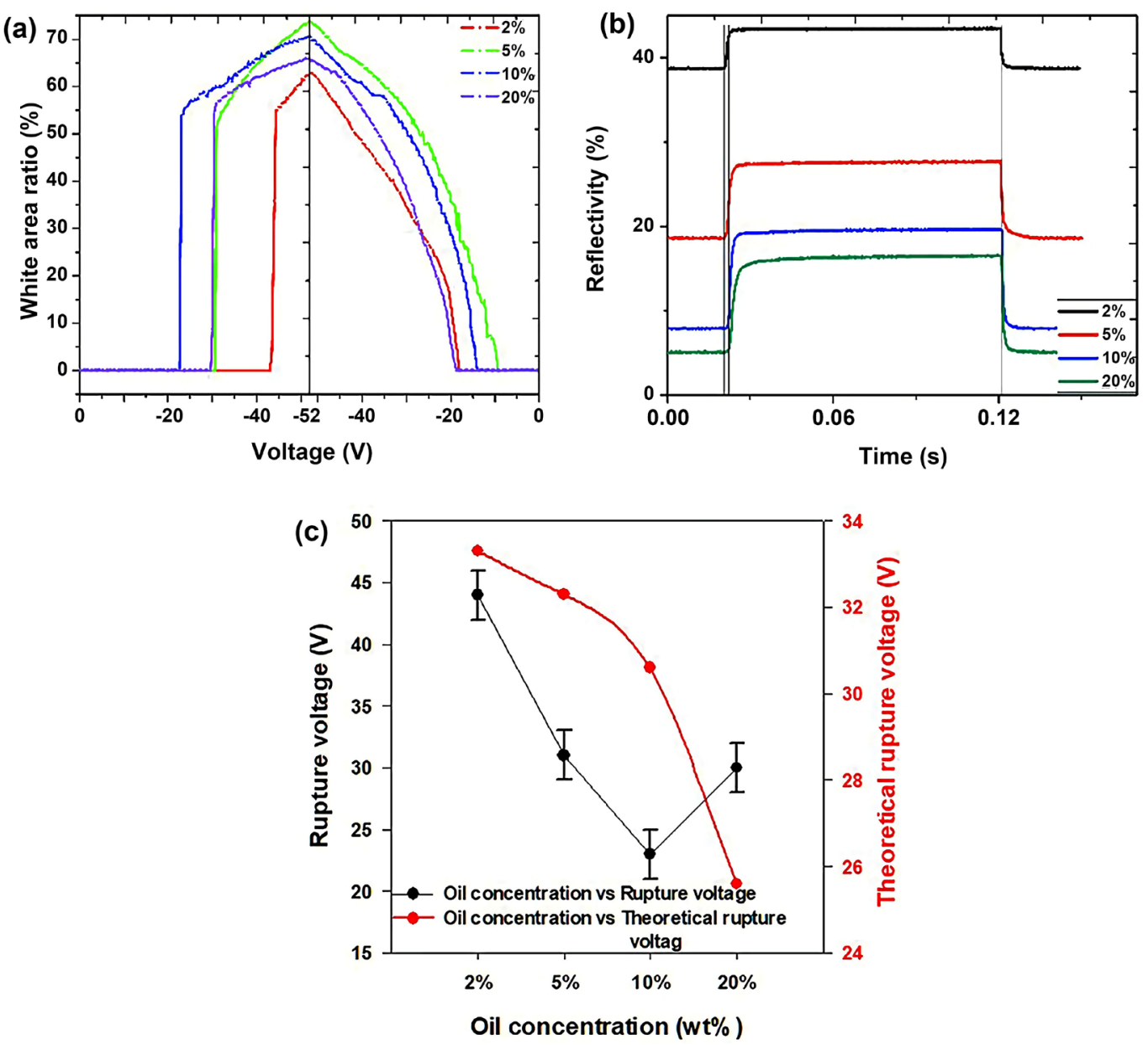

Figure 4. (a) The relation of opening ratio and rupture voltage with oil concentration; (b) the difference of response time of electro fluidic display cell with different concentration oil; (c) Comparision of experimental rupture voltage with the theoretical value.

Figure 4(c) showed the deviation of the theoretical curve and the test data of the rupture voltage. As the interfacial tension is reduced with increased dye concentration, the theoretical curve showed the rupture voltage should decrease, but as Figure 4 (c) shown, as soon as the dye concentration is over $10 \%$ the rupture voltage increases instead of decreasing due to the increased conductivity of the oil phase. The switch-on response time was recorded at $-45 \mathrm{~V}$, which is higher than the rupture voltage to completely open the pixel. According to the literature, the viscosity of the oil solution dominates the dynamics of oil motion after the oil film break [21]. As shown in Figure 3(b), the response time increases with the viscosity of the oil.

\section{Conclusion}

The colored oil was formulated by dissolving an oil-soluble, non-polar purple dye in decane with different concentrations and lauric acid was added to a $2 \%$ dye concentration in decane as an electrolyte. The interfacial tension decreased with increasing oil concentration, but the conductivity increased by a factor of 
10. At lower oil concentration, the interfacial tension was the primary element that influenced the rupture voltage, but when the concentration was higher than $10 \%$, both the conductivity and interfacial tension of oil determined the rupture voltage. The strong polarizability of organic dye molecules under high DC voltage increased the oil solution conductivity. The lauric acid can increase the oil conductivity either by proper ions or by forming inverse micelles in the oil phase. Once the conductivity increases over $10 \mathrm{E}-11$, both the conductivity and the interfacial tension determined the rupture voltage. Higher driving voltage would be needed to achieve the same maximum white area ratio with different oil concentrations due to the increased conductivity. However, the mechanism of charge transfer inside of the oil phase was not fully understood, and no simulation or equation could predict the rupture voltage. The response time was mainly decided by the viscosity of the oil solution.

\section{Acknowledgements}

This work was supported by the National Key R\&D Program of China [2016YFB0401501]; Natural Science Foundation of China under Grant [No. U1601651]; Guangdong Innovative Research Team Program under Grant [No. 2011D039]; Key-Area Research and Development Program of Guangdong Province [2019B010924005]; Science and Technology Program of Guangzhou [No. 2019050001]; Guangdong Provincial Key Laboratory of Optical Information Materials and Technology under Grant [No. 2017B030301007]; MOE International Laboratory for Optical Information Technologies and the 111 Project, Naional Natural Science Foundation of Guangdong China (2018A0303130059).

\section{Conflicts of Interest}

The authors declare no conflicts of interest regarding the publication of this paper.

\section{References}

[1] Lippmann, M. (1875) Relations entre les phénomènes électriques et capillaries. Annales de Chimie et de Physique, 5, 498.

[2] Berge, B. (1993) Electrocapillarity and Wetting of Insulator Films by Water. Comptes Rendus de P Académie des Sciences II, 317, 157-163.

[3] Vallet, M., Vallade, M. and Berge, B. (1999) Limiting Phenomena for the Spreading of Water on Polymer Films by Electrowetting. The European Physical Journal B, 11, 583-591. https://doi.org/10.1007/s100510051186

[4] Quinn, A., Sedev, R. and Ralston, J. (2005) Contact Angle Saturation in Electrowetting. The Journal of Physical Chemistry B, 109, 6268-6275. https://doi.org/10.1021/jp040478f

[5] Pollack, M., Shenderov, A. and Fair, R. (2002) Electrowetting-Based Actuation of Droplets for Integrated Microfluidics. Lab on a Chip, 2, 96-101. https://doi.org/10.1039/b110474h

[6] Rival, D.J.A., Delattre, C., Fouillet, Y., Castellan, G., Bellemin-Comte, A. and Gi- 
drol, X. (2014) An EWOD-Based Microfluidic Chip for Single-Cell Isolation, mRNA Purification and Subsequent Multiplex qPCR. Lab on a Chip, 14, 3739-3749. https://doi.org/10.1039/C4LC00592A

[7] Berge, B. and Peseux, J. (2000) Variable Focal Lens Controlled by an External Voltage: An Application of Electrowetting. The European Physical Journal E, 3, 159-163. https://doi.org/10.1007/s101890070029

[8] Hendriks, B.H.W., Kuiper, S., As, M.A.J.V., Renders, C.A. and Tukker, T.W. (2005) Electrowetting-Based Variable-Focus Lens for Miniature Systems. Optical Review, 12, 255-259. https://doi.org/10.1007/s10043-005-0255-Z

[9] Prins, M.W., Welters, W.J. and Weekamp, J.W. (2001) Fluid Control in Multichannel Structures by Electrocapillary Pressure. Science, 291, 277-280.

https://doi.org/10.1126/science.291.5502.277

[10] Hayes, R.A. and Feenstra, B. (2003) Video-Speed Electronic Paper Based on Electrowetting. Nature, 425, 383-385. https://doi.org/10.1038/nature01988

[11] Beni, G. and Hackwood, S. (1981) Electro-Wetting Displays. Applied Physics Letters, 38, 207-209. https://doi.org/10.1063/1.92322

[12] Decamps, C. and De Coninck, J. (2000) Dynamics of Spontaneous Spreading under Electrowetting Conditions. Langmuir, 16, 10150-10153. https://doi.org/10.1021/la000590e

[13] Roques-Carmes, T., Palmier, S., Hayes, R.A. and Schlangen, L.J. (2005) The Effect of the Oil/Water Interfacial Tension on Electrowetting Driven Fluid Motion. Colloids and Surfaces A, 267, 56-63. https://doi.org/10.1016/j.colsurfa.2005.06.056

[14] Giraldo, A., Massard, R., Mans, J., Derckx, E., Aubert, J. and Mennen, J. (2011) 10.3: Ultra Low-Power Electrowetting-Based Displays Using Dynamic Frame Rate Driving. SID Symposium Digest of Technical Papers, 42, 114-117. https://doi.org/10.1889/1.3621027

[15] Guo, Y., Deng, Y., Xu, B., Henzen, A., Hayes, R., Tang, B. and Zhou, G. (2018) Asymmetrical Electrowetting on Dielectrics Induced by Charge Transfer through an Oil/Water Interface. Langmuir, 34, 11943-11951.

https://doi.org/10.1021/acs.langmuir.8b01718

[16] Gan, S., Chen, L., Feng, Y., Deng, Y., Zhou, R., Dou, Y., Tang, B., Shui, L., Wang, Y. and Li, H. (2018) Protonation-Induced Molecular Permeation at the Oil/Water Interface in an Electric Field. Physical Chemistry Chemical Physics, 20, 29012-29017. https://doi.org/10.1039/C8CP04028A

[17] Zhou, K., Heikenfeld, J., Dean, K.A., Howard, E.M. and Johnson, M.R. (2009) A Full Description of a Simple and Scalable Fabrication Process for Electrowetting Displays. Journal of Micromechanics and Microengineering, 19, Article ID: 065029. https://doi.org/10.1088/0960-1317/19/6/065029

[18] Susana Zeppieri, J.R.G. and Lopez de Ramos, A.L. (2001) Interfacial Tension of Alkane + Water Systems. Journal of Chemical Engineering, 46, 1086-1088. https://doi.org/10.1021/je000245r

[19] Lima, E.R.A., Melo, B.M.D., Baptista, L.T. and Paredes, M.L.L. (2013) Specific Ion Effects on the Interfacial Tension of Water/Hydrocarbon Systems. Brazilian Journal of Chemical Engineering, 30, 55-62. https://doi.org/10.1590/S0104-66322013000100007

[20] Massard, R., Mans, J., Adityaputra, A., Leguijt, R., Staats, C. and Giraldo, A. (2013) Colored Oil for Electrowetting Displays. Journal of Information Display, 14, 1-6. https://doi.org/10.1080/15980316.2012.751939 
[21] Tang, B., Groenewold, J., Zhou, M., Hayes, R.A. and Zhou, G. (2016) Interfacial Electrofluidics in Confined Systems. Scientific Reports, 6, Article No. 26593.

https://doi.org/10.1038/srep26593 